\title{
Network Coded Multicast over Multibeam Satellite Systems
}

\author{
R. Alegre-Godoy and M. A. Vazquez-Castro \\ Department of Telecommunications and Systems Engineering, Engineering School, Autonomous University of Barcelona, \\ 08193 Barcelona, Spain \\ Correspondence should be addressed to R. Alegre-Godoy; ricard.alegre@uab.cat
}

Received 23 February 2015; Accepted 27 May 2015

Academic Editor: Zhen-Lai Han

Copyright ( 2015 R. Alegre-Godoy and M. A. Vázquez-Castro. This is an open access article distributed under the Creative Commons Attribution License, which permits unrestricted use, distribution, and reproduction in any medium, provided the original work is properly cited.

\begin{abstract}
We propose a multicast scheme for multibeam satellite systems exploiting both the multiuser and spatial diversity inherent in this type of systems while taking into account realistic physical distributions of User Terminals (UTs) over the coverage. Our proposed scheme makes use of the well-known Adaptive Coding and Modulation (ACM) feature in Digital Video Broadcasting over Satellite, 2nd Generation (DVB-S2) and Extension (DVB-S2X) standards but also incorporates a set of innovative features. First, multilink reception, that is, receivers that can syntonize different frequencies and/or polarizations, together with Network Coding (NC) is used to enable decoding of signals from adjacent beams (spatial diversity). Second, efficient and fair allocation of resources is achieved through Proportionally Fair Multicast (PFM) scheduling. Our results, obtained over realistic nonuniform UTs distributions, show average system multicast throughput gains up to $88 \%$ with regard to state-of-the-art multicast schemes. Furthermore, a complete cross-layer architecture is proposed, fully compliant with the standard providing Quality of Service (QoS) guarantees.
\end{abstract}

\section{Introduction}

Recent studies are showing an increasing demand for the efficient distribution of personalized contents in Internet based networks [1]. This has led to the deployment of satellite platforms delivering high throughputs (HTS systems) such as Ka-SAT [2] or constellations of communication satellites such as the O3b system [3]. Recent works even consider collocating two satellites covering the same region in order to cope with the user needs $[4,5]$. Beyond the aforementioned satellite physical aspects, it is also possible to satisfy the user needs by improving the logical mechanisms delivering multimedia contents. Multicasting is one of the cornerstones for the effective dissemination and distribution of personalized multimedia contents in broadband networks and the focus of this paper. Applications such as audio/video streaming, online gaming, file distribution, and file downloading are based on multicast-like transmissions.

In wireless networks, including multibeam satellite networks, the main challenge when multicasting is how to address the heterogeneous channels conditions of the User
Terminals (UTs), that is, the presence of multiuser diversity. In clear-sky conditions, the difference in Signal to Interference plus Noise Ratio (SINR) between a UT located at the center of the beam and a UT located at the edge is typically $2-3 \mathrm{~dB}$. When Adaptive Coding and Modulation (ACM) is adopted at the physical layer as in Digital Video Broadcasting over Satellite, 2nd Generation (DVB-S2) [6] and DVB-S2 Extension (DVB-S2X) [7] standards, this difference in SINR is translated into a spectral efficiency difference of $11 \%-25 \%$ $[6,8]$. The differences in spectral efficiency can be much higher if we consider a beam partially affected by a rain event.

Traditional colouring schemes in multibeam systems allow many opportunities for exploiting spatial diversity. A UT can potentially access a number of orthogonal transmissions from the adjacent beams [8]. In that case, UTs in the border of the beam would be more advantaged than those in the center of the beam. Since current UTs are syntonized at a single frequency, orthogonal transmissions are not exploited. A multilink receiver, that is, a receiver able to syntonize different frequencies and polarizations, could access and decode the signal meant for adjacent beams. As mentioned 
in [8], the design of this type of receivers is perfectly possible with the current technology. UTs distribution also plays a fundamental role. In real life, users are not uniformly distributed but concentrated in specific areas such as cities. The concentration of terminals in specific areas of the coverage also affects the performance of cellular based systems $[9,10]$.

In this paper, we take into account these three aspects, multiuser and spatial diversity and UTs distribution to design a multicasting scheme for the efficient delivery of broadband contents.

(a) Related Works on Satellite Multicast. Multicast in multibeam satellite systems has been little investigated from the scheduling and resources allocation point of view. The authors in [11] propose to choose a fixed Reed Solomon code and a fixed rate out of a set of possible rates in order to accomplish a certain degree of reliability. Following a similar approach to [11], works in $[12,13]$ propose to choose a modulation and codification (MODCOD) which ensures reception to a subset of the UTs in the multicast group. The rest could only decode with a certain probability. In [14], a traditional approach is adopted and information is multicasted according to the channel conditions of the worst UT in the multicast group. With respect to these works, our approach takes advantage of multiuser diversity in order to select in each time-slot the optimal MODCOD rather than assuming a fixed scheme. To the best of our knowledge, the authors in [15] provide the most similar approach to our work since the scheme they propose is based on ACM. In particular, authors propose a Network Utility Maximization (NUM) to trade delay and rate also accounting for Quality of Service (QoS) and multiuser diversity. With respect to this work, we introduce the novelty of multilink reception UTs together with Network Coding (NC) which enables decoding orthogonal transmissions.

Furthermore, our paper breaks the traditional approach of assuming uniform UTs distributions and provides results and analysis for nonuniform distributions which are close to reality.

(b) Related Works on NC for Multibeam Satellite Systems. In the past few years, a number of works have studied the implementation of NC in multibeam satellite systems. In [16], an overview of possible satellite scenarios where $\mathrm{NC}$ can be applied is provided. For the particular case of multibeam satellite systems, NC is proposed as a mechanism to reduce retransmissions. Works in $[17,18]$ take advantage of the orthogonal transmissions available using multilink reception. However, the focus is on unicast transmissions and NC is used to provide enhanced reliability and flexibility rather than increasing the throughput. Our previous works in $[19,20]$ assessed the feasibility of using NC for multicasting in multibeam satellite systems. As a result, it was identified that the multilink reception approach together with NC coding could bring important benefits subject to the location of the UTs. These papers describe a preliminary concept and lack of a

(i) method to decide when to use the multilink reception with NC feature, (ii) scheduling policy selecting the optimal MODCOD for the multicast service.

(c) Contributions of the Paper. This paper proposes a full multicasting scheme, that is, scheduling policy, packet scheduling architecture, and algorithm to decide if the multilink reception with NC feature must be used or not. Our work presents the following novel results with respect to use of $\mathrm{NC}$ technology:

(i) A technique for the joint use of multilink reception, NC, and Proportionally Fair Multicast (PFM).

And it also presents the following novel results in the field of satellite multicasting:

(i) Introduce and adapt the PFM scheduling concept in [21]. More specifically, we provide MODCOD selection and use of the multilink reception with $\mathrm{NC}$ feature when suitable.

(ii) Its associated cross-layer packet scheduling architecture with respect to the Internet Engineering Task Force (IETF) differentiated services at IP level model (Diffserv).

(iii) A scheme providing multicast throughput gains employing the same resources as a traditional multicast scheme demonstrated via analysis and simulations over theoretical and realistic nonuniform UTs distributions.

The rest of the paper is organized as follows: Section 2 introduces the multibeam satellite system model. Section 3 describes the proposed multicast scheme. The packet scheduling architecture is introduced in Section 4. Finally, Section 5 provides numerical evaluation of the system performance and Section 6 draws conclusions on the work done.

\section{System Model}

2.1. Multibeam Satellite System Model. We assume a multibeam and multigateway satellite system with $N_{b}$ beams, $P$ polarizations, and frequency reuse factor $f_{r}$. The number of colours of the system is $N_{c}=P f_{r}$. Forward link transmissions are based on DVB-S2/DVB-S2X with ACM. Each gateway $(\mathrm{GW})$ is associated with a subset of the overall number of beams (or cluster). GWs receive channel state information (CSI) messages from the UTs through a feedback channel.

Let a GW of the system serve the subset of beams $\mathscr{C}=$ $\left\{b_{1}, b_{2}, \ldots, b_{\mid \mathscr{C}\}}\right\}$. Each beam $b_{j} \subset \mathscr{C}$ has assigned a number of UTs $u_{j}$ requesting the same multicast service. Let us derive the SINR for a UT $i$ in $b_{j}, 1 \leq i \leq u_{j}$. First, let the number of cochannel beams of the overall system be $K=N_{b} / N_{c}$. Now, we define $\mathbf{H} \in \mathbb{C}^{K \times K}$ as the forward link channel matrix which can be decomposed as $\mathbf{H}=\mathbf{B G}$. Matrix $\mathbf{B} \in \mathbb{C}^{K \times K}$ accounts for the atmospheric, propagation, space, and ground system effects and is defined as

$$
\mathbf{B}=\operatorname{diag}\left(\sqrt{\beta_{1}}, \sqrt{\beta_{2}}, \ldots, \sqrt{\beta_{K}}\right),
$$


where $\beta_{j}=\mathrm{OBO}_{\mathrm{HPA}} L_{\text {sat }} L_{\text {prop }} G_{\mathrm{UT}}$, with $\mathrm{OBO}_{\mathrm{HPA}}$ being the output back-off of the satellite high power amplifier, $L_{\text {sat }}$ the satellite repeater losses, $L_{\text {prop }}$ the propagation losses, and $G_{U T}$ the UT antenna gain. Matrix $\mathbf{G} \in \mathbb{C}^{K \times K}$ accounts for the square root of the satellite antennas gains towards the concrete position of the UT and is defined as

$$
\mathbf{G}=\left(\begin{array}{ccc}
\sqrt{g_{11}} & \cdots & \sqrt{g_{1 K}} \\
\vdots & \ddots & \vdots \\
\sqrt{g_{K 1}} & \cdots & \sqrt{g_{K K}}
\end{array}\right),
$$

where $\sqrt{g_{v w}}$ stands for the square root of antenna gain for antenna $v$ towards the location of the UT at beam $w$. Therefore, each element $h_{v w} \in \mathbf{H}$ accounts for all the gains and losses from satellite antenna $v$ towards UT location at beam $w$. The received signal $y_{i, j}$ at UT $i \in b_{j}$ can be expressed as

$$
\begin{aligned}
y_{i, j}(x, y)= & \sqrt{P_{\text {sat }}} h_{j j}(x, y) s_{j}+\sqrt{P_{\text {sat }}} \sum_{l \neq j} h_{l j}(x, y) s_{l} \\
& +n_{i},
\end{aligned}
$$

where $P_{\text {sat }}$ is the satellite transmitted power, $n_{i}$ is the Gaussian noise (zero mean complex circular noise of variance $N_{i}$ ), and $s_{j}$ and $s_{l}$ are the transmitted and interfering symbols, respectively. Assuming constant transmitting power, the SINR can be extracted directly from (3) and is given by

$$
\Gamma_{i, j}(x, y)=\frac{P_{\text {sat }}\left|h_{j j}(x, y)\right|^{2}}{P_{\text {sat }} \sum_{l \neq j}\left|h_{l j}\right|^{2}+N_{i}} .
$$

Under the ACM specification of DVB-S2 and DVB-S2X, SINR values are mapped to spectral efficiencies (or equivalently MODCODs) as follows:

$$
\begin{aligned}
& \eta_{i, j}=f_{\text {DVB-S2 }}\left(\Gamma_{i, j}\right), \\
& \eta_{i, j}=f_{\text {DVB-S2X }}\left(\Gamma_{i, j}\right),
\end{aligned}
$$

where $\eta_{i, j}$ is the spectral efficiency for UT $i$ in $b_{j}$ and $f_{\text {DVB-S2}}, f_{\text {DVB-S2X }}$ are mapping functions that relate SINRs and spectral efficiencies for DVB-S2 and DVB-S2X standards, respectively.

2.2. Multilink Reception System Model. The work in [8] introduces and models multilink multibeam systems. Such systems assume the use of multilink receivers, that is, receivers which can syntonize different frequencies or polarizations to simultaneously decode orthogonal transmissions from adjacent beams.

The main concept is as follows. In a multibeam system with $N_{c}$ colours, UTs can potentially decode up to $N_{c}$ transmissions, 1 transmission from the own beam and $N_{c}-1$ transmissions from adjacent beams in orthogonal frequencies and/or polarizations. This effect is produced because the antenna gain of each spot-beam is so high, that even UTs outside of the beam observe values of SINR that lie within the range of available MODCODs and can decode the signal. To do so,

(i) UTs must have multilink reception capabilities, for example, a terminal with a single antenna, one Low Noise Block downconverter (LNB), and multiple reception chains to detect and decode different polarizations and bands;

(ii) UTs must observe a value of SINR higher than or equal to the one required to decode the MODCOD transmitted in the orthogonal beam. Conversely, the GW can lower the MODCOD transmitted in a beam to let a number of UTs outside of the beam decode the signal.

In our multicasting scheme, we assume that UTs can decode their own transmission and one out of the $N_{c}-1$ orthogonal transmissions, more specifically, the transmission with strongest SINR or equivalently the transmission from the closest adjacent beam. This enables an extra path to reach each UT and the opportunity to exploit spatial diversity. Let subscript $a$ denote the adjacent beam whose signal intends to decode UT $i$ in $b_{j}$ and let $\Gamma_{i, j}^{a}(x, y)$ be the SINR observed from this adjacent beam. $\Gamma_{i, j}^{a}(x, y)$ is obtained particularizing (4) with beam $a$, that is, substituting $h_{j j}$ with $h_{a j}$ and computing the interference power from the cochannel beams of $a$. The spectral efficiency achievable from such adjacent beam is denoted as $\eta_{i, j}^{a}$ and obtained particularizing (5) with $\Gamma_{i, j}^{a}(x, y)$.

Figure 1 shows the MODCODs achievable for different locations (in $U / V$ coordinates [22]) within a beam of a 70-beam system. More specifically, the top plot shows, per each location, the adjacent beam providing better SINR (the location and the adjacent beam with the strongest SINR are plotted in the same color and dashed lines separate the different areas). The mid and bottom plots show the achievable MODCODs from the own and determined adjacent beam transmissions, respectively. It can be observed that any point of the beam can decode an orthogonal transmission and that locations close to the edge of the beam and in the beam overlapping areas can decode it with a high order MODCOD. Locations in the center of the beam could only decode signals employing low spectral efficiency MODCODs.

2.3. Network Coding Model. In our multicast scheme, UTs can simultaneously decode their own transmission and one orthogonal transmission. The objective of $\mathrm{NC}$ is to avoid receiving duplicated versions of the same packet and ease the scheduling at the GW side. Therefore, whenever our proposed scheme sends packets over two paths, those packets will be coded employing NC. We perform NC at the IP layer for the following reasons:

(i) Our objective is to increase the average multicast throughput in a single multicast service. IP allows identifying the content of the packets and classifying them into different multicast services such that $\mathrm{NC}$ is performed in a intraservice manner. Working at lower layers would not allow this classification. 

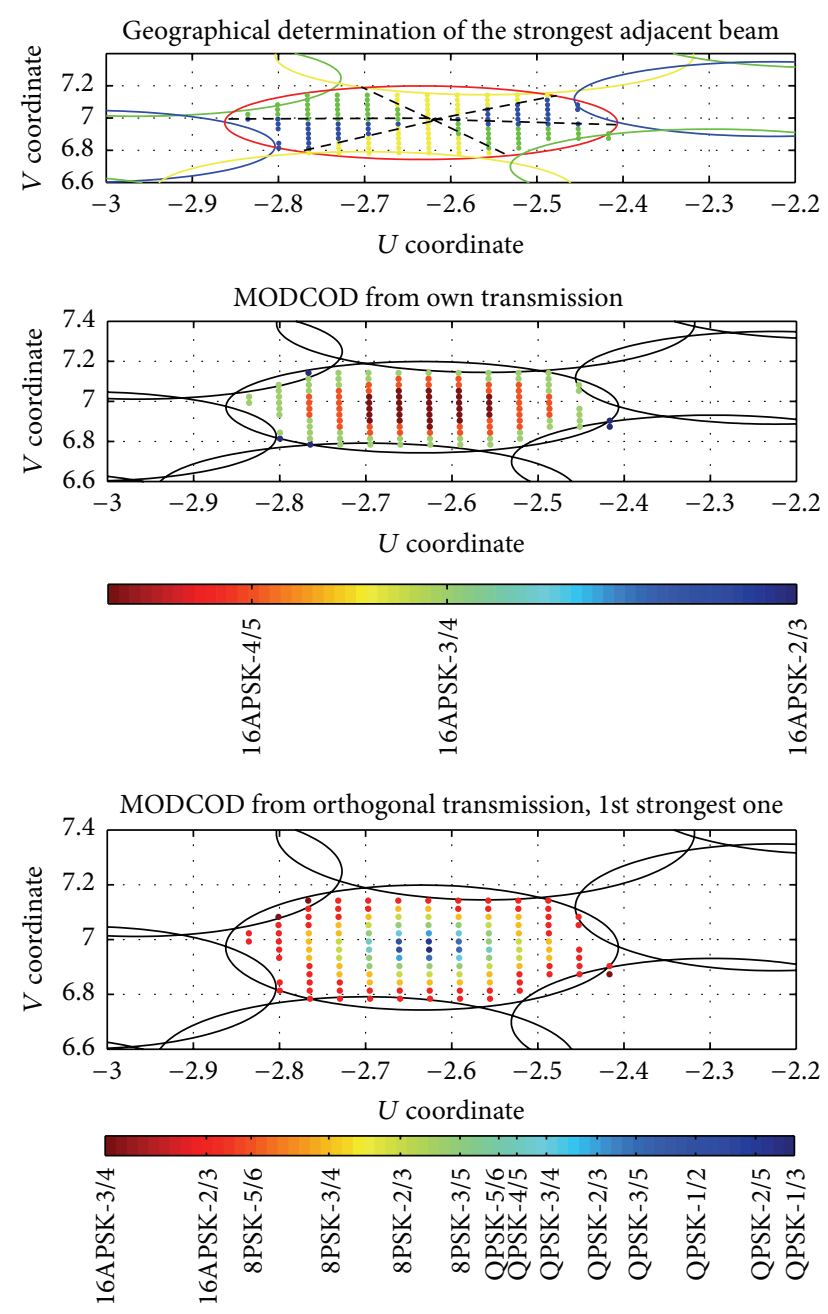

FIGURE 1: Example of achievable MODCODs for locations within a beam of interest. Results extracted from a 70-beam, 4-coloured system under DVB-S2.

(ii) IP headers can be easily modified to include the encoding coefficients necessary for decoding [23].

In particular, we adopt Random Linear Network Coding (RLNC) due to its simplicity and capacity-achieving performance, although any other network code would be, in principle, applicable. The scheme generates a random linear coded packet from $N_{p}$ native packets as follows. Let $L$ denote the length in bits of a native IP packet. Then, the payload of each packet $p_{t}$ is split into blocks of $\epsilon$ bits. Let $d_{t k}$ denote the $k$ th block of $t$ th packet, with $1 \leq k \leq L / \epsilon$. Next, we choose $N_{p}$ random coefficients $c_{t}$ from $\mathbb{F}_{q}$, with $q=2^{\epsilon}$. The $k$ th coded block of a coded packet is given by

$$
C_{k}=\sum_{t=1}^{N_{p}} c_{t} d_{t k}, \quad 1 \leq k \leq \frac{L}{\epsilon} .
$$

The encoding coefficients $c_{1}, \ldots, c_{N_{p}}$ are added in the header of the packet unless pseudorandom codes are used and then only the seed needs to be sent. In the receiver side, at least $N_{p}$ coded packets must be received to retrieve the original packets. The parameter $\epsilon$ is the size of the finite field and should be big enough to ensure that the probability of generating two linearly dependent (l.d.) coded packets is negligible. Sections 3 and 4 introduce the specific parameters and architectural details necessary to perform RLNC.

2.4. User Terminals Distribution. We employ the method in [24] to generate the nonuniform distributions of UTs. Specifically, we break the area of interest, that is, the cluster of beams $\mathscr{C}$, in a number of bins $N_{z}$. Each bin $z$ is assigned a different probability $P_{z}$ of containing a UT according to a function $\phi_{z}$. In this case, $\phi_{z}$ is a function of the coordinates $x_{z}, y_{z}$ of the bin and given by a symmetrical 2D truncated Gaussian function [9]:

$$
\phi_{z}\left(x_{z}, y_{z}\right)=\alpha \frac{\exp \left(-\alpha\left[\left(x_{z}-x_{0}\right)^{2}+\left(y_{z}-y_{0}\right)^{2}\right]\right)}{\pi\left[1-\exp \left(-\alpha R_{0}^{2}\right)\right]},
$$

where $x_{0}, y_{0}$ is the UTs distribution center, $R_{0}$ is the radius, and $\alpha$ is the location index. To express $\phi_{z}$ as a probability, it must be normalized by the total sum of $\phi_{z}$ over all the $N_{z}$ bins considered:

$$
P_{z}\left(x_{z}, y_{z}\right)=\frac{\phi_{z}\left(x_{z}, y_{z}\right)}{\sum_{z=1}^{N_{z}} \phi_{z}\left(x_{z}, y_{z}\right)}
$$

Then, we can model UTs distribution in a cluster $\mathscr{C}$ by the quadruple $\left(\alpha, R_{0}, x_{0}, y_{0}\right)$ and analyze average multicast throughput results varying these parameters. Negative values of $\alpha$ concentrate UTs towards the edge of the cluster whilst positive values of $\alpha$ concentrate UTs around $x_{0}, y_{0} . \alpha=0$ provides a uniform distribution.

\section{Multicast Proportional Fairness for Multibeam Satellite Systems}

Figure 2 shows a functional diagram of the proposed multicast scheme based on a cross-layer architecture. Novel blocks, at GW and UT side, are shadowed in green. We define multicast as the transmission of a data flow (or multicast service) from the GW to a number of UTs in the cluster $\mathscr{C}$ controlled by the GW. The UTs requesting the service are known as the multicast group. The scheme we propose applies to each multicast service individually (intraservice), and hence explanations of the multicast policies assume a single multicast service. Scheduling is performed on a slot-by-slot basis; that is, in the current slot we choose how packets are to be transmitted in the next slot. The scheme has been designed such that if follows the coherence time of the channel (i.e., time windows in the scheduling are not required because the satellite channel for fixed UTs shows only medium/long term variations even under rain conditions).

On the GW side, we introduce 3 novel blocks which carry out the Link Layer (LL) scheduling and substitute classical scheduling policies. Let us assume a system with ACM at the physical layer and let $M$ be the number of MODCODs of the system. We denote by $\eta^{m}$ the spectral efficiency of the $m$ th 


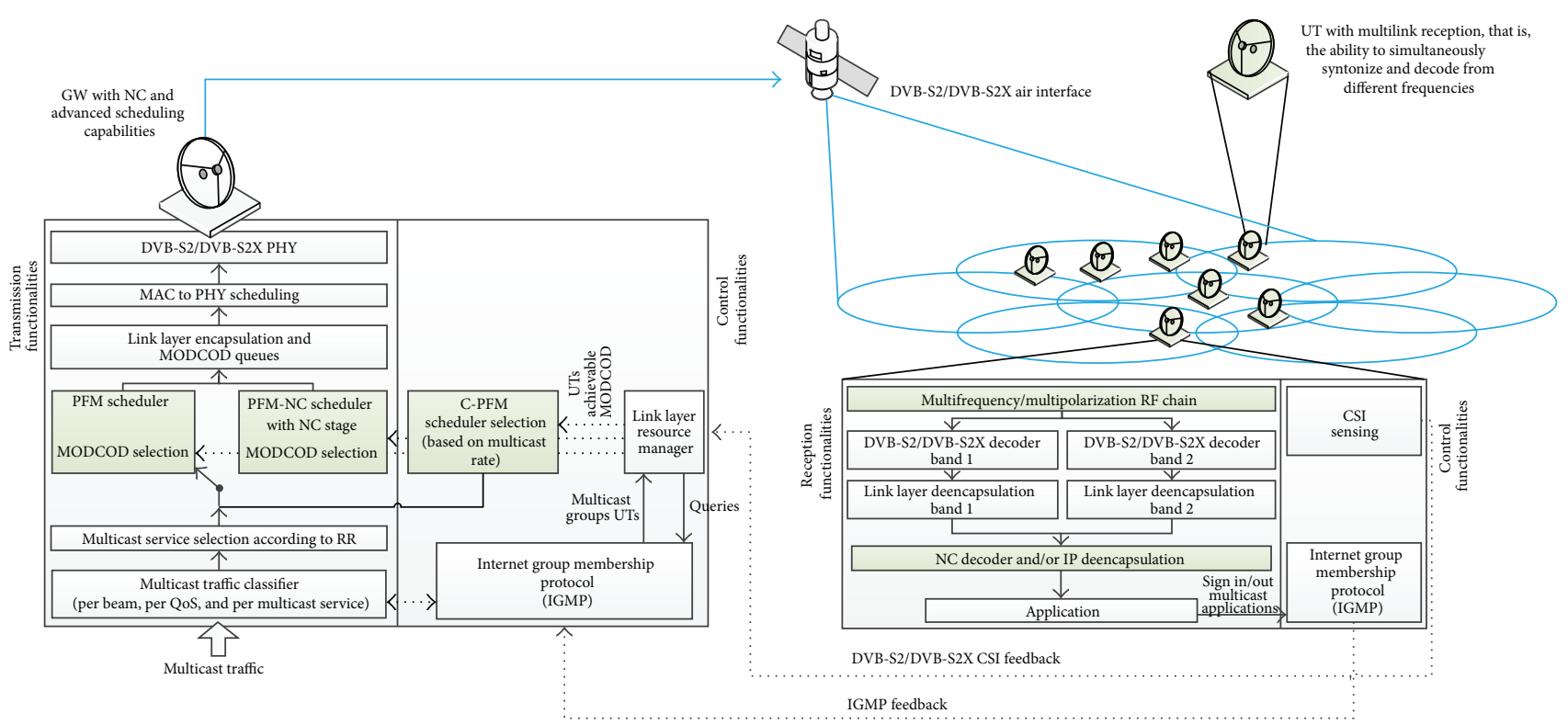

FIGURE 2: Functional diagram of the proposed multicast scheme based on a cross-layer architecture.

MODCOD, with $1 \leq m \leq M$. The bitrate provided by $\eta^{m}$ is given by

$$
r^{m}=R_{s} \eta^{m}
$$

where $R_{s}$ denotes the system symbol rate. The objective of LL scheduling, formed by the 3 green blocks, is twofold: (1) Choose the most appropriate transmission mode in each time-slot; that is, decide whether the multilink reception capabilities of the UTs have to be exploited or not. (2) Choose the appropriate value of spectral efficiency (i.e., MODCOD) for the next time-slot according to the transmission mode selected and the CSI reports received from the UTs in the current slot. This is equivalent to say the multicast policy is scheduling a certain number of UTs (those able to decode the selected MODCOD). In particular,

(i) the block Proportionally Fair Multicast (PFM) computes per each beam of the cluster $\mathscr{C}$ (and per multicast service) which is the most suitable MODCOD according to the proportionally fair rule, and hence it exploits multiuser diversity;

(ii) the block PFM with NC (PFM-NC) computes for the entire cluster $\mathscr{C}$ a common MODCOD for all the beams of the cluster delivering that service; data is sent network coded using RLNC and UTs use its multilink reception capabilities to attempt decoding the signal from its own beam and its adjacent beam with higher SINR;

(iii) the block combined PFM (C-PFM) selects the most appropriate transmission mode, PFM (nonuse of multilink reception capabilities), or PFM-NC (use of multilink reception capabilities) in each time-slot by computing the mode obtaining the highest multicast rate; (iv) the inputs required for the PFM, PFM-NC, and CPFM blocks are the MODCODs achievable by the UTs in base to their CSI feedback. The LL Resource Manager (LLRM) provides this information to these 3 blocks.

PFM, PFM-NC, and C-PFM blocks are described in detail in Sections 3.1, 3.2, and 3.3, respectively. The rest of the GW follows a traditional approach such that our proposed scheme can be implemented via software modifications with minimal intrusion. Incoming multicast traffic is classified per beam, per QoS following the IETF Diffserv model, and per multicast service before being forwarded to the LL schedulers. The specific packet scheduling architecture of the GW is described in detail in Section 4.

The membership management of the multicast groups is supported by the Internet Group Management Protocol (IGMP) [25]. The LLRM collects information of the multicast groups available at the IGMP block and the CSI of the UTs. UTs not only send the CSI with respect to its own beam but also the CSI with respect to all its adjacent beams. The collaboration between the IGMP block and the LLRM provides the following information: (1) which beams are involved in the transmission of a certain multicast service; (2) the number of UTs per beam and its SINR with respect to the own and adjacent beams; (3) combining (1) and (2), identifying the orthogonal transmission with the strongest SINR.

On the UT side, the RF chain must be able to syntonize two different bands (frequencies and/or polarizations) in order to obtain the packets from the own beam and from the strongest adjacent beam. Physical layer and link layer packets are deencapsulated in both bands up to the IP layer. At the IP layer, the UT checks if packets are network coded by looking at the IP headers: 
(i) If not, packets from the adjacent beam are discarded and the packets from the own beam are deencapsulated as usual and forwarded to the upper layers.

(ii) If yes, decoding of the network coded packets is performed before deencapsulating and forwarding the packets to the upper layers.

Although this may appear as a complex receiver, the multilink reception does only need a single antenna and a single LNB as explained in Section 2.2. Even if multiple reception chains to detect and decode different bands are necessary, such kind of terminals already exists and is defined for instance in Digital Video Broadcasting Satellite to Handheld (DVB-SH) for mode B devices [26]. DVB-S2X also provides tools to implement transmission of a single stream through several physical satellite transponders and how to carry out its reception through several DVB-S2X decoders in the receiver (the so-called channel bonding for multitune receivers [7]). With regard to the decoding of network coded packets, a number of works have successfully assessed the feasibility of the technology in broadband networks; see, for instance, [2729].

3.1. Proportionally Fair Multicast (PFM). In this subsection, we introduce the PFM block in Figure 2. In a PF allocation, UTs with better channel state are scheduled more often than UTs with worse channel states. To this aim, the GW performs the scheduling in two steps. (1) Compute the optimal perbeam MODCOD for the next time-slot. (2) Update the scheduled rates of each UT according to the MODCOD selected in Step (1) for the next iteration.

Step 1. The MODCOD chosen for the next time-slot at beam $b_{j}$ out of the $M$ available MODCODs is given by

$$
\begin{array}{r}
m_{j}^{p f}(t+1)=\arg \max _{m}\left\{\prod_{i \mid r_{i, j}(t) \geq R_{s} \eta^{m}}\left(1+\frac{R_{s} \eta^{m}}{R_{i, j}(t)}\right)\right\} \\
\forall j \in[1,|\mathscr{C}|],
\end{array}
$$

where $R_{i, j}(t)$ accumulates the scheduled rates of UT $i$ in $b_{j}$ and $r_{i, j}(t)$ is the achievable bitrate by UT $i$ in $b_{j}$ given by $r_{i, j}(t)=$ $R_{s} \eta_{i, j}(t)$.

Step 2. Let $\mathcal{S}_{j}^{p f}(t+1)$ denote the set of UTs in $b_{j}$ which will be scheduled at $t+1$ and given by

$$
\delta_{j}^{p f}(t+1)=\left\{i \mid r_{i, j}(t) \geq r^{m_{j}^{p f}}(t+1)\right\}
$$

where $r^{m_{j}^{p f}}(t+1)=R_{s} \eta^{m_{j}^{p f}}(t+1)$ is the bitrate provided by spectral efficiency $\eta^{m_{j}^{p f}}(t+1)$ at beam $b_{j}$. Subsequently, $R_{i, j}$ is updated by

$$
R_{i, j}(t+1)= \begin{cases}R_{i, j}(t)+r^{m_{j}^{p f}}(t+1), & i \in S_{j}^{p f}(t+1) \\ R_{i, j}(t), & \text { elsewhere }\end{cases}
$$

The PFM scheme takes advantage of multiuser diversity because (10) selects the most appropriate MODCOD per time-slot according to the channel conditions across the UTs.

\subsection{Proportionally Fair Multicast with Network Coding (PFM-}

$N C$ ). We now introduce the PF block employing NC (PFM-

NC) in Figure 2 to take advantage of the extra path provided by orthogonal transmissions. To this aim, the GW considers the cluster as one entity with a number of UTs $U=\sum_{j=i}^{|\mathscr{C}|} u_{j}$ and performs the scheduling in 3 steps. (1) Select for each UT the orthogonal transmission with the strongest SINR from which it will receive packets in addition to those sent through its own beam. (2) Compute the optimal MODCOD to use in the cluster for the next time-slot taking into account orthogonal transmissions. (3) Update the scheduled rates of each UT according to the MODCOD selected in Step (2) for the next iteration.

Since UTs receive packets from two paths employing the same MODCOD, the min-cut/max-flow theorem states that up to 2 different packets can be received simultaneously. Due to the layout of the multibeam network, the only way to ensure that all scheduled UTs will receive both packets is to send packets network coded. For instance, Figure 3(a) shows an example where one of the UTs would only receive one of the packets $p_{1}$ and $p_{2}$ independently of the packets sent by each queue. Instead, if every queue generated a single coded packet, all UTs would receive $p_{1}$ and $p_{2}$. Moreover, NC simplifies the scheduling because the system just transmits combined packets instead of looking at the topology of the network and decides which packets should be selected in each queue for transmission; for example, Figure 3(b) shows that all UTs can obtain $p_{1}$ and $p_{2}$ but previous knowledge of the topology is necessary to decide which packet forwards each queue. Hence, under PFM-NC, the per-beam queues of a specific multicast service will generate a single coded packet from the same $N_{p}=2$ native packets as explained in Section 2.3. Then, all the UTs able to decode the chosen MODCOD will always get two different coded packets. Those UTs unable to decode orthogonal transmissions will get only one coded packet and will not be able to convey the original packets; that is, they are considered as nonscheduled UTs.

Step 1. Select for each UT an adjacent beam, that is, the orthogonal transmission with strongest SINR, in order to decode simultaneously from two different paths. Such adjacent beam is chosen as follows. Let $\mathscr{A}_{j}=\left\{a_{1}, \ldots, a_{\left|\mathscr{A}_{j}\right|}\right\}$ be the set of adjacent beams to $b_{j}$ and let $\Gamma_{i, j}^{a_{e}}$ be the SINR of UT $i$ in $b_{j}$ with respect to the adjacent beam $a_{e}$. UT $i$ in $b_{j}$ selects the adjacent beam $a$ such that

$$
e^{*}=\arg \max _{e}\left\{\Gamma_{i, j}^{a_{e}} \mid a_{e} \in \mathscr{A}_{j}, a_{e} \in \mathscr{C}, u_{e} \neq 0\right\} .
$$

The related spectral efficiency of $\Gamma_{i, j}^{a_{e^{*}}}, \eta_{i, j}^{a_{e^{*}}}$, is obtained from (5). The conditions in the argmax in (13) restrict the selection of $a_{e}$ to those adjacent beams that belong to $\mathscr{C}$ and have at least one UT subscribed to the multicast service. Note that this differentiates our scheme from load balancing techniques since we do not use sparing resources from an adjacent beam 


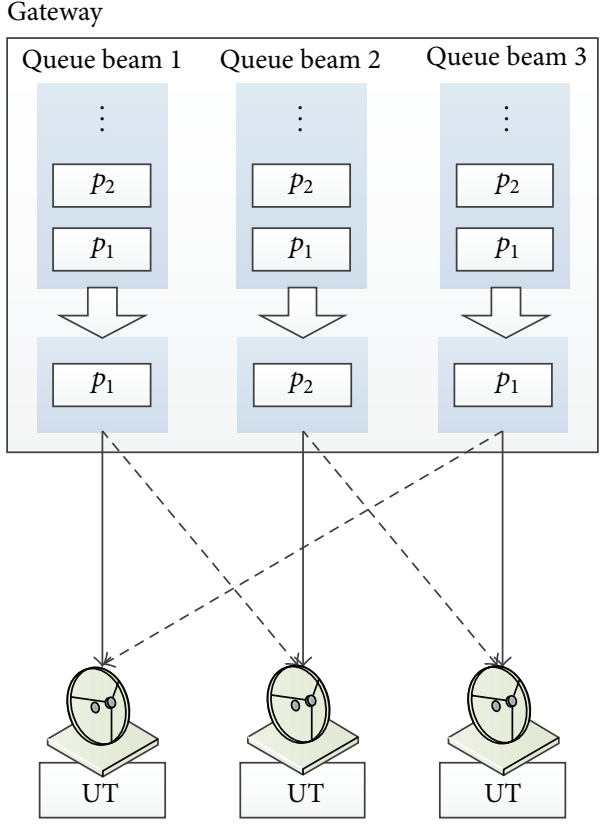

(a)

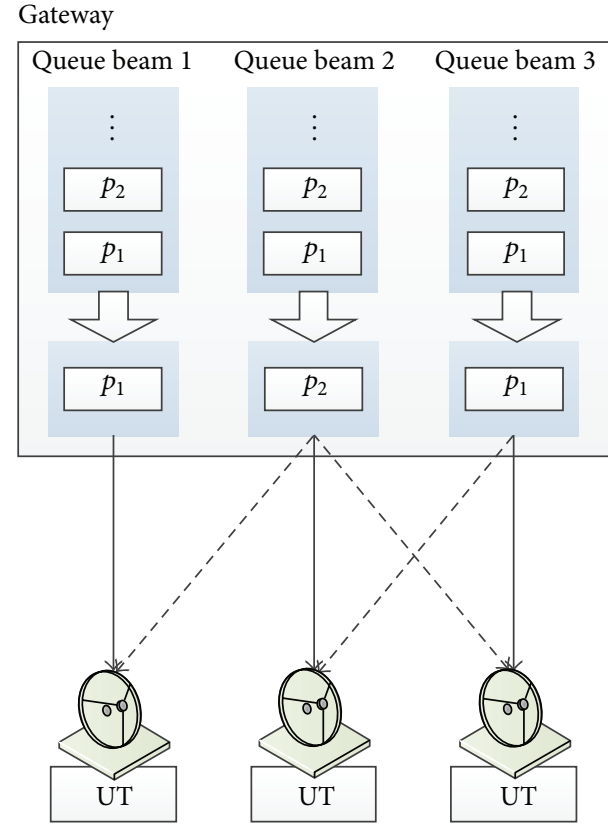

(b)

FIGURE 3: Two examples of inefficient multicasting from a GW transmitting uncoded packets to three UTs in three beams. Continuous and dashed arrows represent transmissions from the own and best adjacent beam, respectively.

but we take advantage of the resources already in use in the adjacent beam. If none of the adjacent beams accomplish the conditions in (13) or the SINR from the chosen adjacent beam lies without the MODCOD range, then $\eta_{i, j}^{a_{e^{*}}}=0$.

Step 2. The optimal MODCOD in the PFM-NC scheme is given by

$$
m^{n c}(t+1)=\arg \max _{m}\left\{\prod_{i \mid r_{i, j}^{a_{j}^{*}}(t) \geq R_{s} \eta^{m}}\left(1+\frac{2 R_{s} \eta^{m}}{R_{i, j}(t)}\right)\right\},
$$

where $r_{i, j}^{a_{e}^{*}}(t)=R_{s} \eta_{i, j}^{a_{e}}$. Thus, given a value of $R_{s} \eta^{m}$, the product in (14) takes into account only those UTs that can decode at such rate from the orthogonal transmission. Implicitly, this means that the UT can support that rate from the own transmission and therefore factor 2 in the numerator of (14).

Step 3. Let $S^{n c}(t+1)$ denote the set of UTs in $\mathscr{C}$ scheduled at $t+1$ and given by

$$
S^{n c}(t+1)=\left\{i \mid r_{i, j}^{a_{e}^{*}}(t) \geq r^{m^{n c}}(t+1)\right\},
$$

where $r^{m^{n c}}(t+1)=R_{s} \eta^{m^{n c}}(t+1)$ is the bitrate provided by spectral efficiency $\eta^{m^{n c}}$ in the cluster $\mathscr{C}$. Subsequently, $R_{i, j}$ is updated by

$$
R_{i, j}(t+1)= \begin{cases}R_{i, j}(t)+2 r^{m^{n c}}(t+1), & i \in S^{n c}(t+1) \\ R_{i, j}(t), & \text { elsewhere. }\end{cases}
$$

When a UT is scheduled, we take into account that it receives information from two paths by adding a factor of 2 in the second term of the scheduled rates.

3.3. Combined PFM/PFM-NC (C-PFM). In the two previous subsections, we have introduced the two blocks of Figure 2 based on the PF rule, PFM, and PFM-NC. In short, (1) PFM exploits multiuser diversity. Under clear-sky conditions, it tends to favour UTs in the center of the beam. (2) PFM-NC exploits multiuser and spatial diversity. Under clear-sky conditions, it tends to favour UTs in the edge of the beam. Following, we introduce the control block to select the most appropriate scheme for transmitting in each time-slot, that is, the selection block named C-PFM. Let $f_{j}^{p f}\left(\eta^{m}\right)$ denote the product in (10) and let $f^{n c}\left(\eta^{m}\right)$ denote the product in (14). $f_{j}^{p f}\left(\eta^{m}\right)$ and $f^{n c}\left(\eta^{m}\right)$ evaluate the average multicast rates at a given $\eta^{m}$ for the PFM and PFM-NC schemes, respectively. Hence, at a given time-slot, we compute $m_{j}^{p f}(t+1)$ and $m^{n c}(t+1)$ and evaluate the scheme providing better average multicast rate by comparing the outputs of $f_{j}^{p f}\left(\eta^{m_{j}^{p f}}(t+1)\right)$ and $f^{n c}\left(\eta^{m^{n c}}(t+1)\right)$. Since the latter takes into account all the UTs in the cluster, we perform the comparison with the product of all $f_{j}^{p f}\left(\eta^{m_{j}^{p f}}(t+\right.$ $1)$ ). Then, scheduled rates must be updated according to the scheme selected using (12) or (16). Algorithm 1 shows the CPFM multicasting scheme we propose.

\section{Packet Scheduling Architecture}

In this section, we describe the packet scheduling architecture for the proposed multicast scheme. Although our work 
(1) At time-slot $t$ obtain $m_{j}^{p f}(t+1) \forall j$ and $m^{n c}(t+1)$ based on (10) and (14) respectively.

(2) Compute $f_{j}^{p f}\left(\eta^{m_{j}^{p f}}(t+1)\right) \forall j$ and $f^{n c}\left(\eta^{m^{n c}}(t+1)\right)$.

(3) if $\prod_{j=1}^{|\mathscr{C}|} f_{j}^{p f}\left(\eta^{m_{j}^{p f}}(t+1)\right) \geq f^{n c}\left(\eta^{m^{n c}}(t+1)\right)$ then

(4) Transmit in PFM mode with MODCOD $m_{j}^{p f}(t+1) \forall j$

(5) Update rates according to (12)

(6) else

(7) Transmit in PFM-NC mode at MODCOD $m^{n c}(t+1)$

(8) Update rates according to (16)

(9) end if

(10) Next time slot

Algorithm 1: C-PFM.

focuses on how to improve the average multicast throughput of a single multicast service, we show how our scheme integrates with an architecture offering more services. We build upon the architecture provided in [30] for unicast transmissions and optimize it for multicast transmissions. Our scheme requires modifications only at the LL level of the scheduler and can be implemented by modifying the software/firmware of the GW. As in [30], QoS between the different multicast services is achieved adopting the IETF Diffserv model at IP level which is a well-known and prevailing satellite QoS provision model [31, 32]. Specifically, 3 Classes of Service ( $\mathrm{CoS}$ ) are defined: Expedited Forward (EF) for premium class services, Assured Forward (AF) for less constrained traffic, and Best Effort (BE) for services with very low or without QoS. Figure 4 shows the proposed packet scheduling architecture for the EF CoS. The structure would be analogous for the AF and BE CoS. The cross-layer control functionalities introduced in Section 3 (see Figure 2) are not shown in the figure to ease the understanding.

Let us assume that the $\mathrm{GW}$ of cluster $C$ transmits $\sigma_{\mathrm{EF}}$, $\sigma_{\mathrm{AF}}$, and $\sigma_{\mathrm{BE}}$ multicast services per $\mathrm{EF}, \mathrm{AF}$, and $\mathrm{BE} \mathrm{CoS}$, respectively. First of all, incoming multicast traffic is classified per beam, per QoS, and per multicast service resulting in a number of IP queues $\sigma_{\mathrm{EF}}, \sigma_{\mathrm{AF}}$, and $\sigma_{\mathrm{BE}}$ per CoS. After that, per each CoS, a coordinated Round Robin (RR) stage pulls packets from a specific service within each CoS. Without loss of generality, let us assume that packets from EF CoS service 1 are pulled. At this point, C-PFM computes, from the UTs achievable MODCODs, which is the optimal transmission mode according to Algorithm 1:

(i) If PFM is selected, the optimal MODCOD per each beam of the cluster $\mathscr{C}$ is computed according to (10). Then, per each beam area, a packet from service 1 is pulled, encapsulated using Generic Stream Encapsulation (GSE) or Multiprotocol Encapsulation (MPE), and forwarded to the appropriate MODCOD queue.

(ii) If PFM-NC is selected, for the entire cluster $\mathscr{C}$, a single optimal MODCOD is computed according to (14). Now, per each beam area, two packets from service 1 are pulled and coded into a single packet using RLNC. This packet is encapsulated using GSE or MPE and forwarded to the appropriate MODCOD queue.
TABLE 1: System characteristics.

\begin{tabular}{lc}
\hline Parameter & Value \\
\hline$N_{b}$ & 70 \\
$N_{c}$ & 4 \\
Frequency & $19.95 \mathrm{GHz}$ \\
System symbol rate & $180 \mathrm{Mbauds}$ \\
PHY protocol & DVB-S2X \\
LL protocol & GSE \\
Network layer protocol & IP \\
IP packets size & 1344 bytes \\
NC overhead & 10 bytes \\
Number of services & 1 \\
\hline
\end{tabular}

GSE/MPE packets are buffered in the MODCOD queues. The Tuneable Fairness Weighted Round Robin (TF-WRR) scheduler in [30] is substituted by a simple First come first served (FCFS) packet selector. Whilst the TF-WRR in [30] allows unicast sessions within a $\operatorname{CoS}$ to be fairly treated, this concept is already provided for each multicast session by our combination of PFM, PFM-NC, and C-PFM blocks. In the last stage before the DVB-S2/DVB-S2X modulator, the Adaptive Weighted Round Robin (AWRR) scheduler provides the QoS guarantees among the different CoS.

\section{Simulation Results}

In order to show results for the proposed scheme, we focus on clusters of $6 / 7$ beams under the control of a single GW within a system of the characteristics of Table 1 . Note that this is a feasible approach since HTS systems require several GW feeder links in order to provide UTs with the required throughputs for broadband and multimedia applications [33, 34]. The UTs distributions in the cluster are generated as detailed in Section 2.4 and for realistic case in Spain. The main figure of merit we analyze is the average system multicast throughput defined as the time average bitrate sum offered to the UTs. Since our scheme applies to multicast services individually and with the purpose of not masking the actual results of the proposed scheme, we focus on the performance of a single multicast performance. The performance of our proposed 


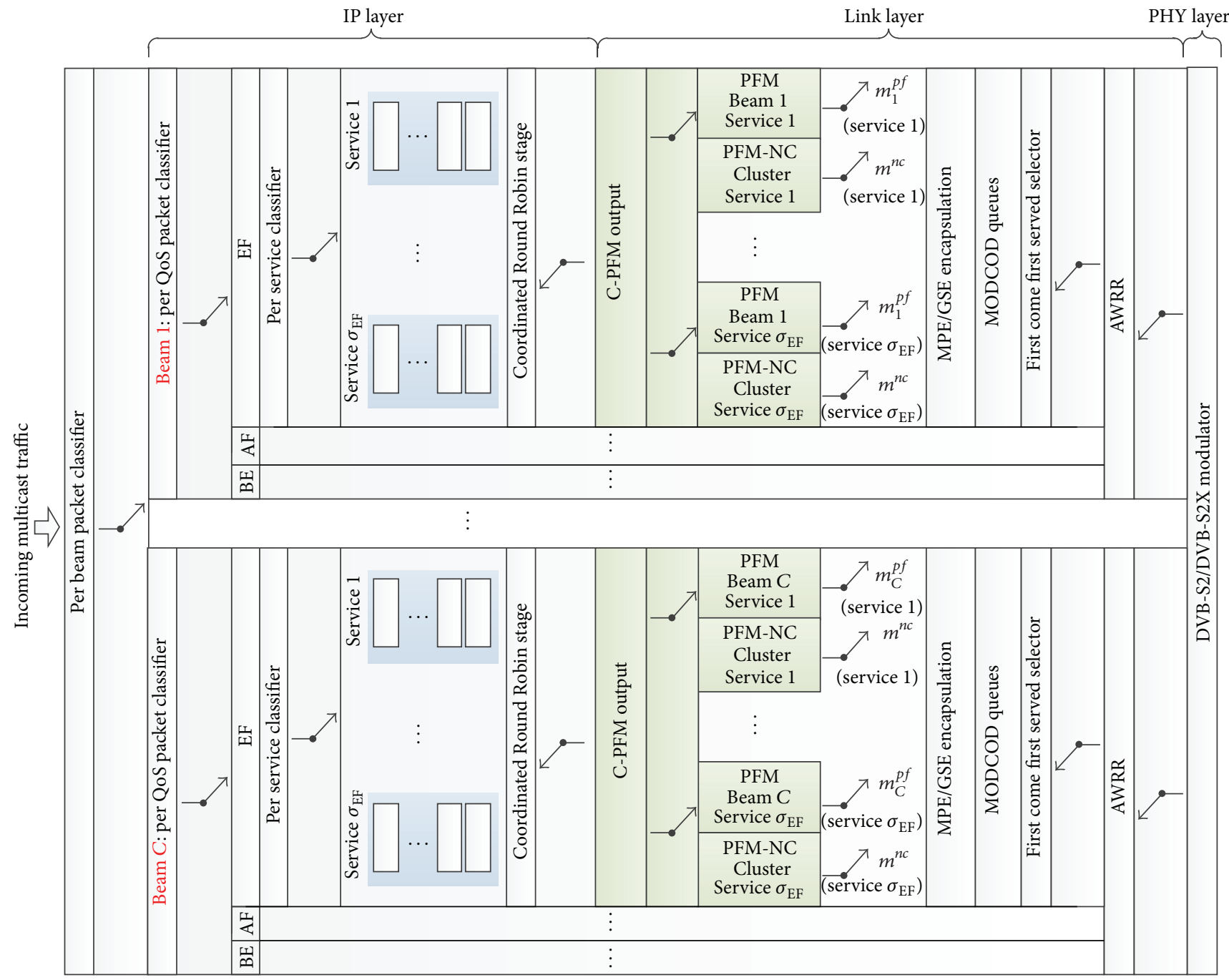

FIGURE 4: Proposed packet scheduling architecture according to IETF Diffserv model at IP level.

scheme is compared to the Worst Case Multicast (WCM) scheme [14] and compared to a scheme with only the PFM implementation (Section 3.1) such that the improvements of the multilink reception with $\mathrm{NC}$ can be easily observed. In the following subsections, we justify the use of DVB-S2X for our particular scheme, provide simulation results for clearsky and rainy conditions, and give practical insights on how to provide a Service Level Agreement (SLA) for UTs that are not so frequently scheduled.

5.1. DVB-S2 versus DVB-S2X. In Figure 1 we have shown, per each location within a beam, the achievable MODCODs when decoding signals from the own and from the adjacent beam providing better SINR for a DVB-S2 based system. Figure 5 shows the equivalent information for a DVB-S2X based system. It can be observed that at the same locations the UTs under DVB-S2X can be assigned MODCODs with higher spectral efficiencies (a combination of higher modulation order and higher code rate) from the own and adjacent beam providing better SINR and hence an improved throughput can be delivered.
Moreover, DVB-S2X incorporates natively the aforementioned channel-bonding tool for multitune receivers to split and uplink a single stream into several satellite transponders (i.e., different beams) and to receive it with a DVB-S2X receiver implementing several physical layers. Hence, DVBS2X seems to be a more appropriate standard to support our scheme.

5.2. Clear-Sky Conditions. Figures 6 and 7 show the average multicast throughput for 6 relevant UTs distributions, generated as in Section 2.4, for a cluster $\mathscr{C}$ of 7 beams.

When UTs tend to be distributed over a large area, as in Figure 6, the proposed C-PFM scheme can achieve gains in average system multicast throughput up to the $27 \%$. Such advantage is obtained when most of the UTs are located in, or around, the overlapping area of the beam because the scheme enables the transmission with multilink reception and NC. Note that the PFM scheme, which does not incorporate multilink reception, attains a similar throughput to the WCM scheme. As most of the UTs tend to be located further from the overlapping area, that is, close to the beam edge or in 

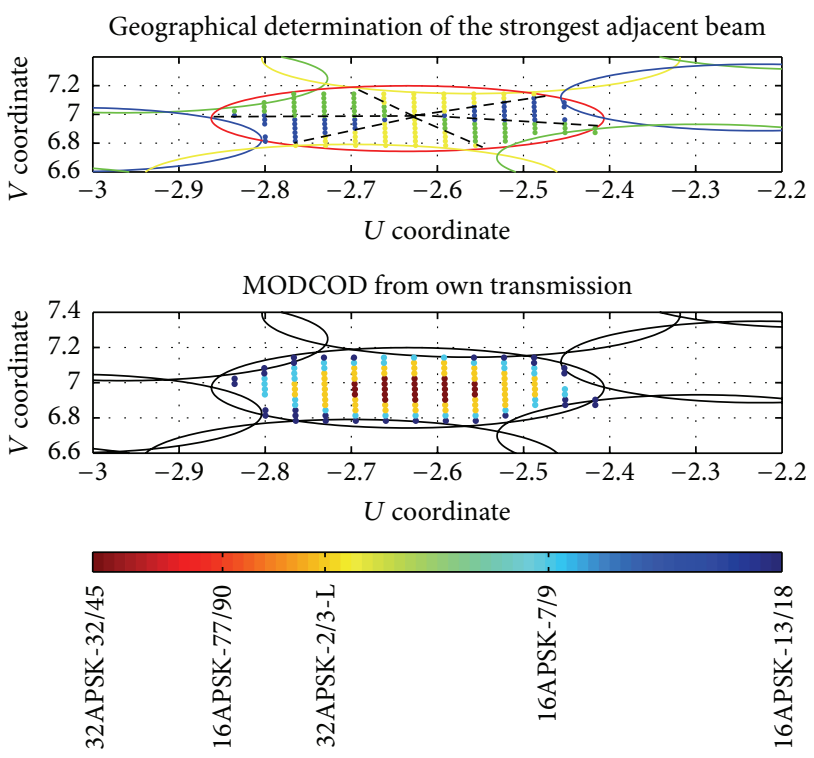

MODCOD from orthogonal transmission, 1st strongest one
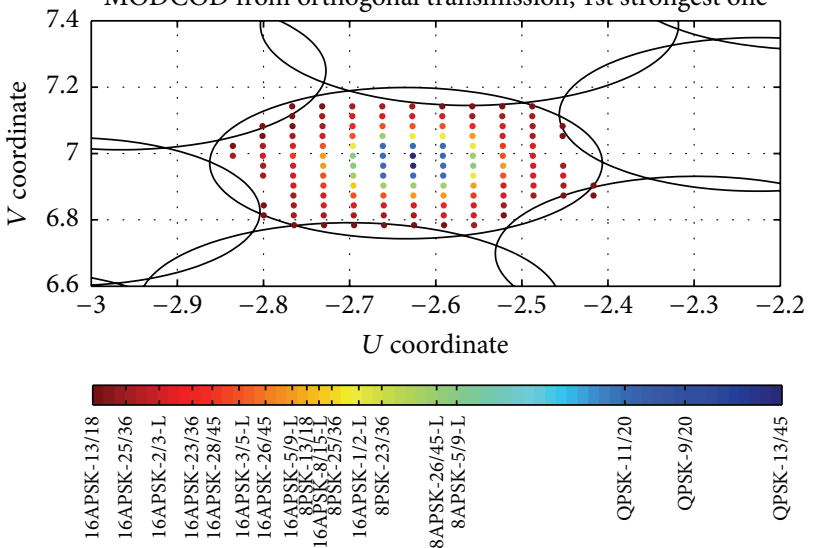

FIgURE 5: Example of achievable MODCODs for locations within a beam of interest. Results extracted from a 70-beam, 4-coloured system under DVB-S2X.

the beam center, the gain obtained decreases to $16 \%$ and $3 \%$, respectively. This is mainly produced because the multilink reception capability with $\mathrm{NC}$ is not activated that often since it is not favourable to the UTs located in the center of the beam. Even if some MODCOD balancing is achieved due to the $\mathrm{PF}$ rule, it is not high enough to provide significant average system multicast throughput gains.

When UTs are concentrated in smaller areas, as Figure 7 shows, the gains tend to be larger. If the UTs are located in the beam overlapping area, the gain in average system multicast throughput is $88 \%$ and it gradually decreases to $64 \%$ and $5 \%$ when UTs are highly concentrated close to the edge of the beam or in the beam center, respectively. Ideally, if all the UTs were located in the beam overlapping area (e.g., in a regional satellite broadcast for a city), a 100\% throughput gain could be achieved, except for the 10 bytes overhead introduced when including the encoding coefficients in the network coded IP packets.

Hence, the proposed C-PFM scheme provides higher gains when UTs are located in the beam overlapping area or close to the edge of the beam concentrated in small $(64 \%-$ $88 \%)$ or large areas $(16 \%-27 \%)$. When UTs are located in the center of the beam, the gain is marginal (3\%-5\%). Moreover, this gain is achieved without employing additional resources, by just enabling UTs accessing orthogonal transmissions already in place.

5.3. Service Level Agreement. The proposed C-PFM scheme optimizes the average multicast throughput of the system according to the PF rule but it does not guarantee a minimum throughput to each individual UT. This side effect of the $\mathrm{PF}$ rule is reflected in the right bottom plot of Figure 7 where the worst UT is assigned a negligible throughput; that is, an SLA is not guaranteed to the UT. Many multicast applications are based on the delivery of video and audio such as video streaming, audio streaming, or online gaming. The SLA guarantees that a minimum amount of data is delivered to the UT such that these services are delivered properly in a similar way traditional Digital Subscriber Line (DSL) operators guarantee a minimum connection quality to users in disfavoured areas while users in better areas get better connection quality.

The results in Figure 8 consider a GW providing multicast services to Spain. The number of UTs subscribed to the multicast service is proportional to the number of inhabitants of the 5 largest Spanish metropolitan areas [35]. We consider a minimum SLA with the UT equivalent to half of the throughput achieved with the highest common achievable MODCOD from the own beam (see top plot of Figure 5); that is, in average each UT should be scheduled once every 2 slots with such MODCOD. As it can be observed in the right bottom plot of Figure 8, without an SLA mechanism such reference level is not reached (the blue bars mark the minimum throughput per UT and the worst UT under the CPFM scheme does not overcome it). A simple mechanism to provide a minimum SLA consists in periodically introducing slots with a MODCOD decodable by all the UTs in the cluster. In particular, the throughput assigned to the worst UT is averaged for a past number of slots and in case it does not reach the proposed level, a MODCOD decodable by such UT is imposed. As it can be observed, now the worst UT is guaranteed the SLA but the average multicast throughput of the cluster has been affected since at certain slots the optimal MODCODs are not scheduled. In the case under study, the provision of SLA has reduced the average system multicast throughput gain compared to WCM from $32 \%$ to $21.5 \%$.

The fact that each transmission is decodable by a different number of UTs due to the use of ACM should not affect the proper delivery of multicast services proved that proper techniques are employed at the application layer. For audio and video streaming and online gaming Scalable Video Coding (SVC) [36] and Scalable Audio Coding (SAC) [37] techniques can be used. For instance, Figure 9 shows the spectral efficiency of the scheduled MODCODs when the SLA is guaranteed. When the MODCOD decodable by all the UTs is transmitted, a video/audio base layer (BL) could be sent such that all the UTs retrieve it. When MODCODs decodable only by a subset of the UTs are transmitted, 

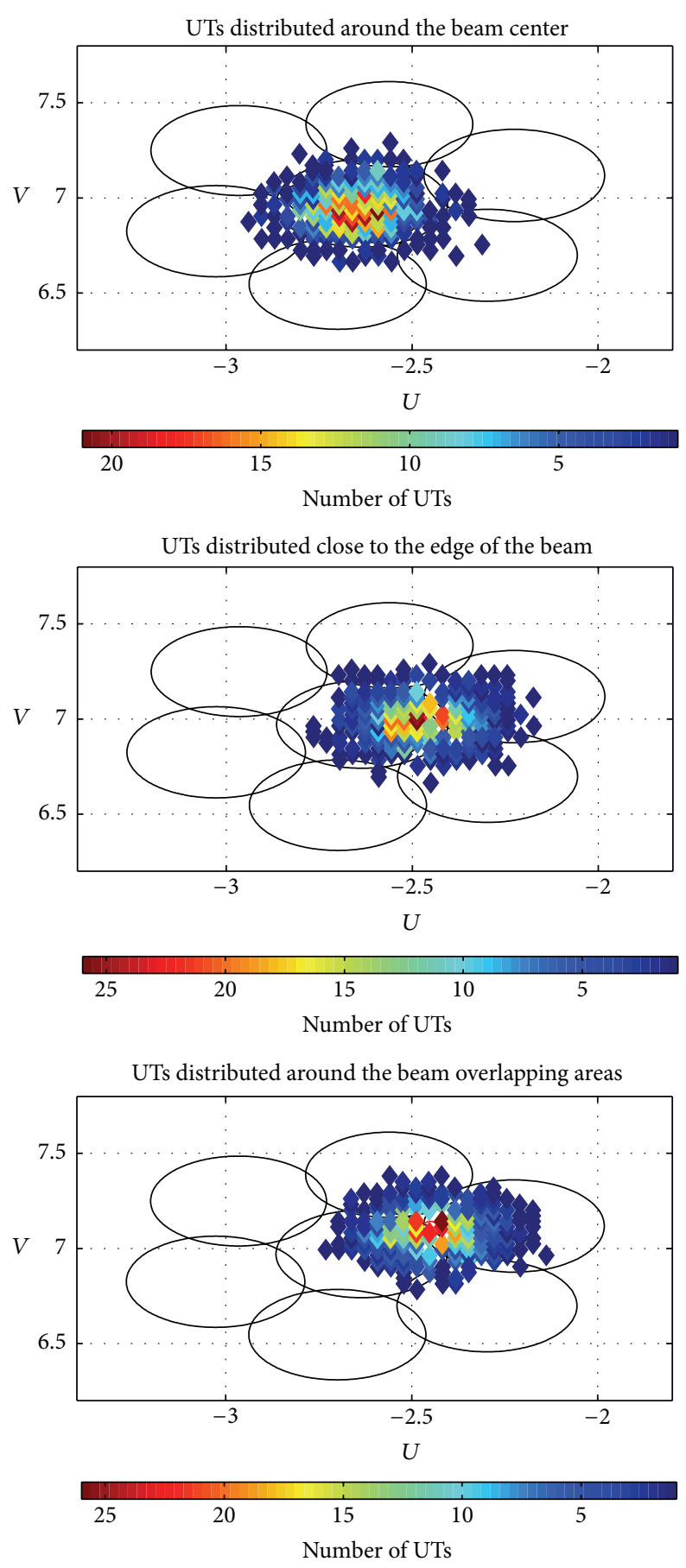

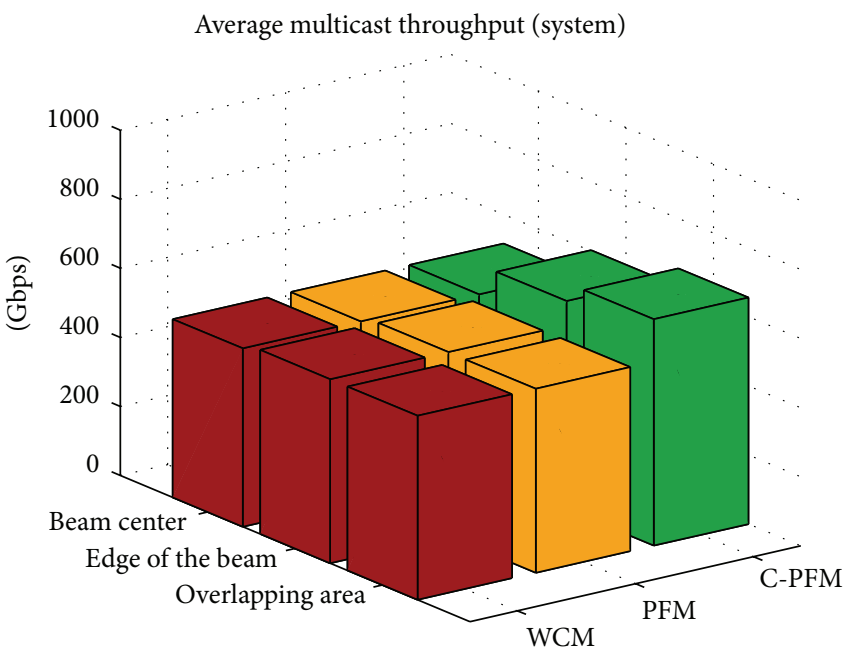

Average multicast throughput (best and worst UT)

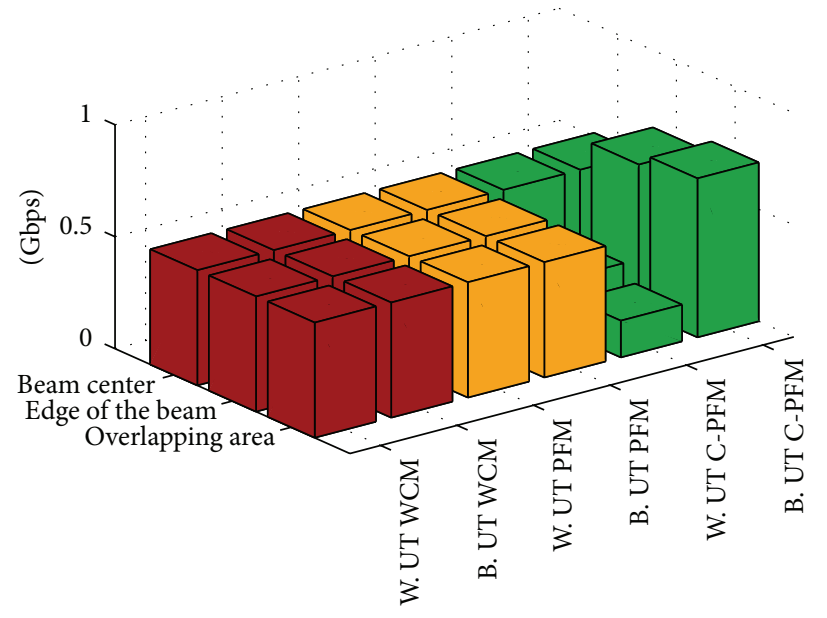

Figure 6: Average multicast throughput for 3-UT distribution within a cluster of beams; $\alpha=50, R_{0}=R_{\mathscr{C}}$, and $x_{0}, y_{0}=\{$ beam center, close to the edge, overlapping area\}.

different levels of video/audio enhancement layers (ELs) can be sent.

For other multicast applications like file distribution and downloading which are less time sensitive, UTs able to decode all the MODCODs would retrieve the files faster and then abandon the multicast group. Then, the scheme would adapt the transmitted MODCODs according to this new set of UTs which could complete the download of the file a little later in time.
5.4. Rain Conditions. Finally, we show that our scheme is also advantageous under rainy conditions while it is able to maintain the SLA guarantee to the UT. To this aim, we assume that the southeast coast of Spain within the dotted ellipse in the left bottom plot of Figure 8 is under rain event. Such event produces a $5 \mathrm{~dB}$ attenuation. As it can be observed in the right bottom plot of Figure 8, the C-PFM scheme still maintains a $15 \%$ gain in average multicast throughput compared to the WCM scheme while keeping the proposed SLA. 

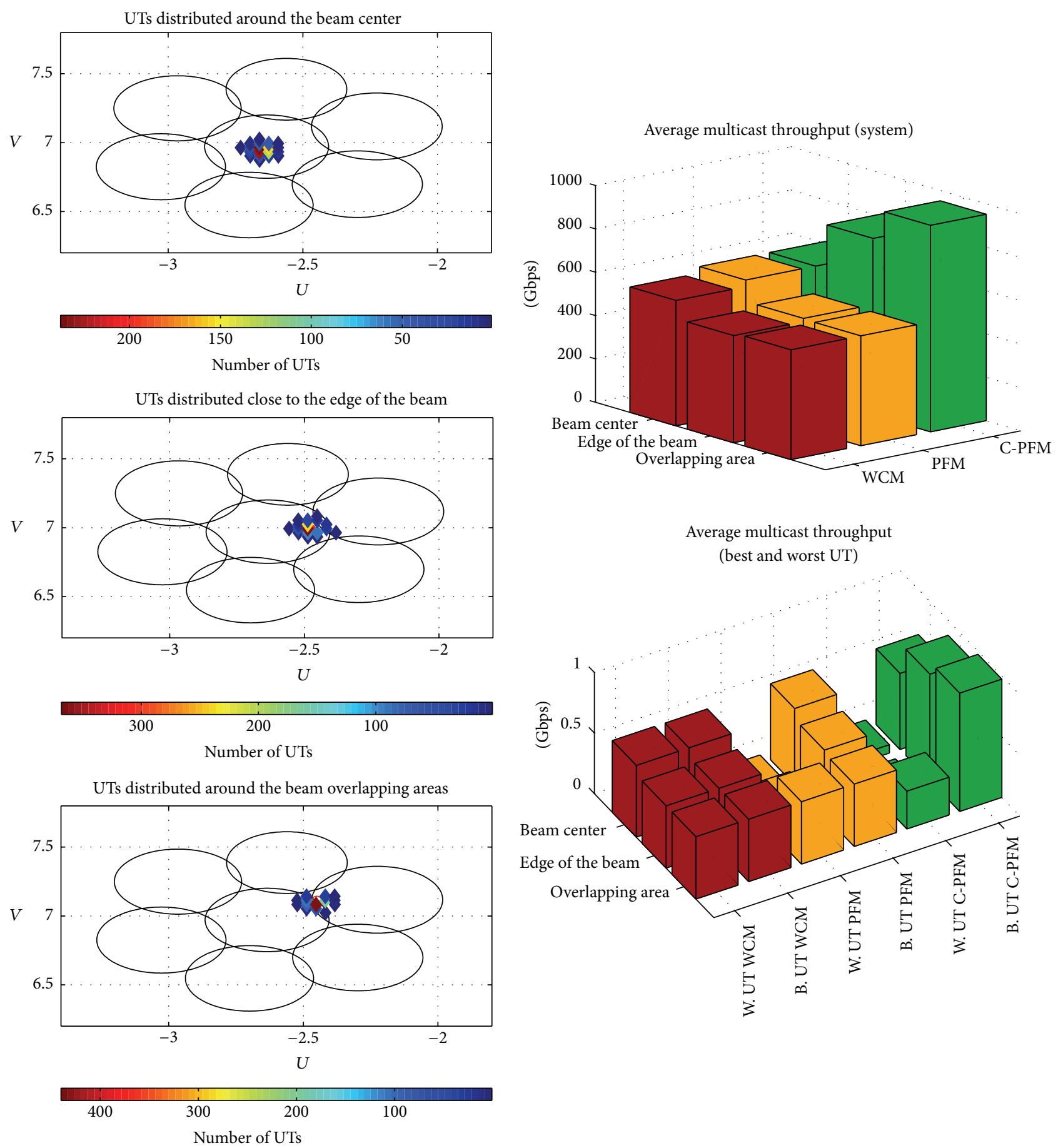

Average multicast throughput (best and worst UT)

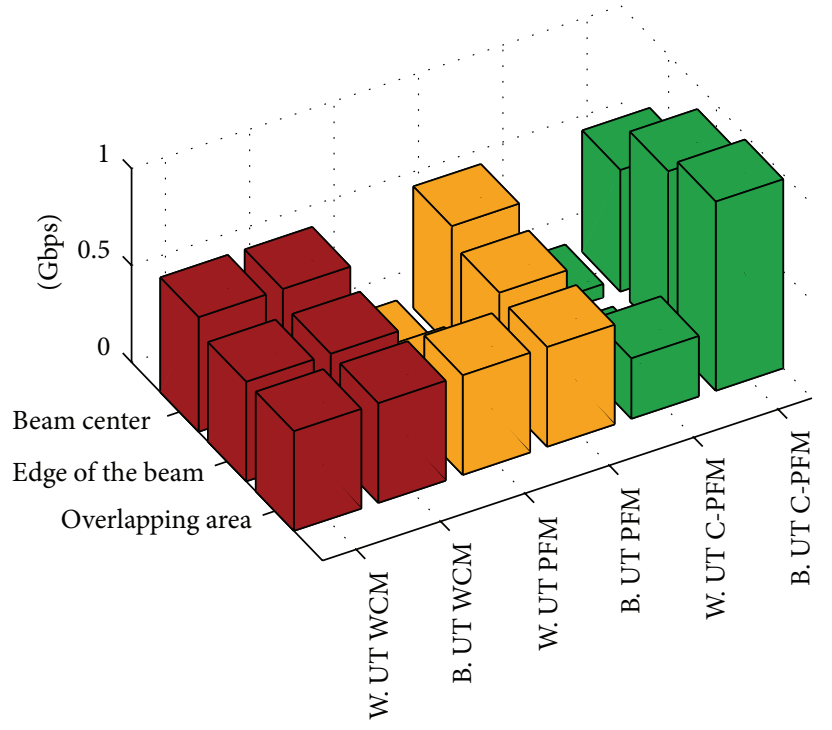

Figure 7: Average multicast throughput for 3-UT distribution within a cluster of beams; $\alpha=1000, R_{0}=R_{\mathscr{C}}$, and $x_{0}, y_{0}=\{$ beam center, close to the edge, overlapping area\}.

\section{Conclusions}

In this paper we have proposed a full multicasting scheme for multibeam satellite systems. Our scheme makes use of the ACM feature of DVB-S2 and DVB-S2X standards by employing a PF scheduling policy and opportunistically enables UTs, assumed to have multilink reception capabilities and a
NC decoder, to retrieve information from the adjacent beam providing better SINR. In particular, we have introduced the functional architecture logic in the GW, based on a cross-layer design, and UT side. We have also detailed the algorithms employed in the novel blocks in the GW and we have designed the packet scheduling architecture respecting the IETF Diffserv model at the IP level. 

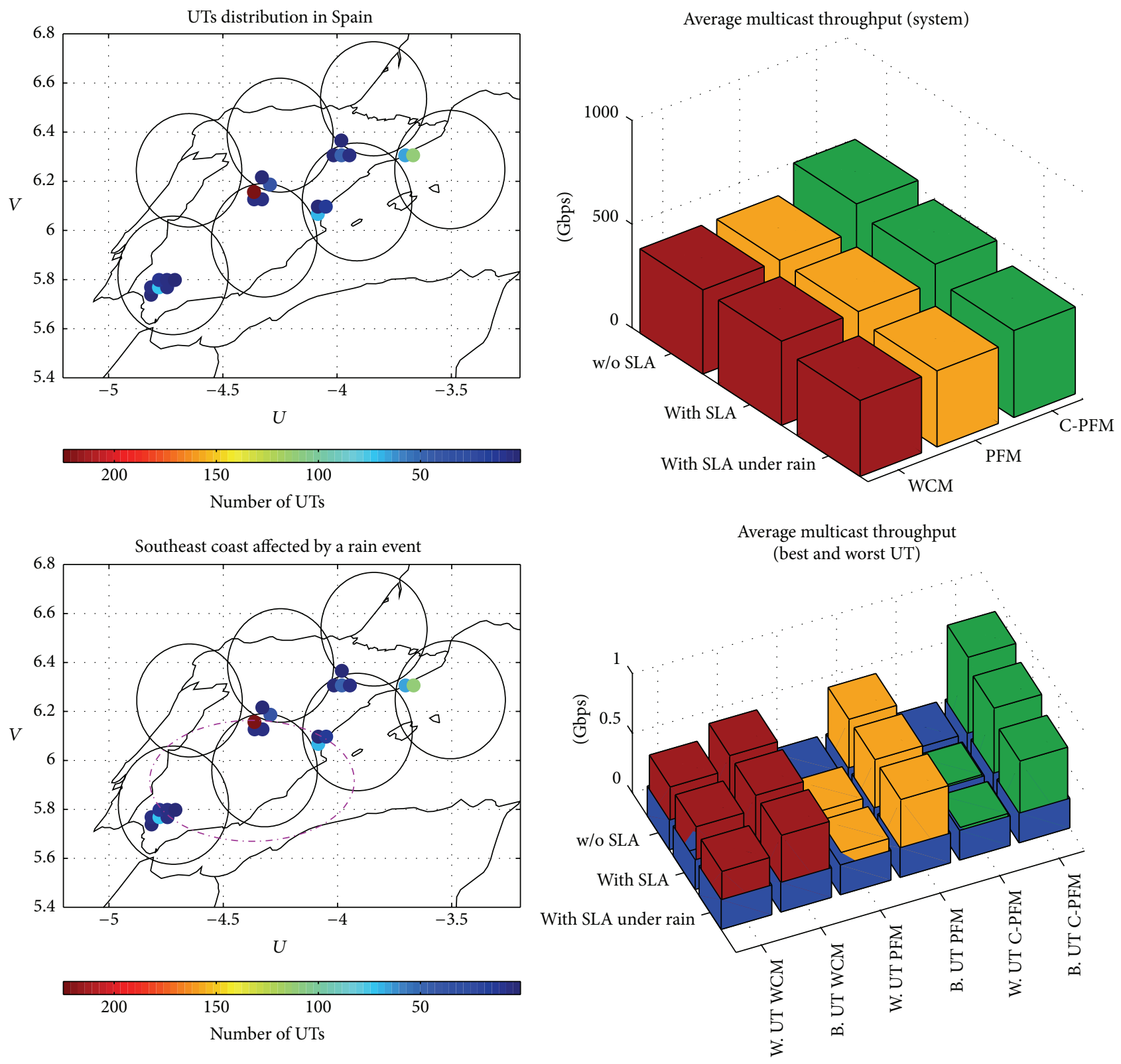

FIGURE 8: Average multicast throughput for realistic UTs distribution in Spain.

From the complexity point of view, the practical introduction of our scheme requires modifications at both the GW and the UT. While modifications in the GW can be implemented via software upgrades, the satellite UT requires further modifications. The RF chain must be able to syntonize two different bands in order to receive from the own and adjacent beam. Also, two separate decoding/deencapsulating chains are necessary up to the IP level. Finally, the UTs need a NC decoder. Although these elements may build up an apparent complex receiver, we have demonstrated that the standards and technology for its implementation are already in place.

From the results point of view, the average system multicast throughput gain provided by our scheme highly depends on the UTs distribution. With the aim of providing fair and realistic results, our simulations have been carried out over nonuniform theoretical and realistic distributions within clusters of few beams where transmissions employ the recently appeared DVB-S2X standard. The results obtained show the following. When the UTs tend to be located in the centers of the beams, the gain provided by our scheme is marginal, that is, below 5\%. However, when most of the UTs are located close to the edge of a beam or in the overlapping areas with another beam, even if the UTs spread over a large area of the cluster, the gain provided by our scheme can reach the $88 \%$. This gain is mainly enabled by the multilink reception and NC.

Moreover, a simple SLA mechanism has been introduced and simulated such that a minimum service is guaranteed 


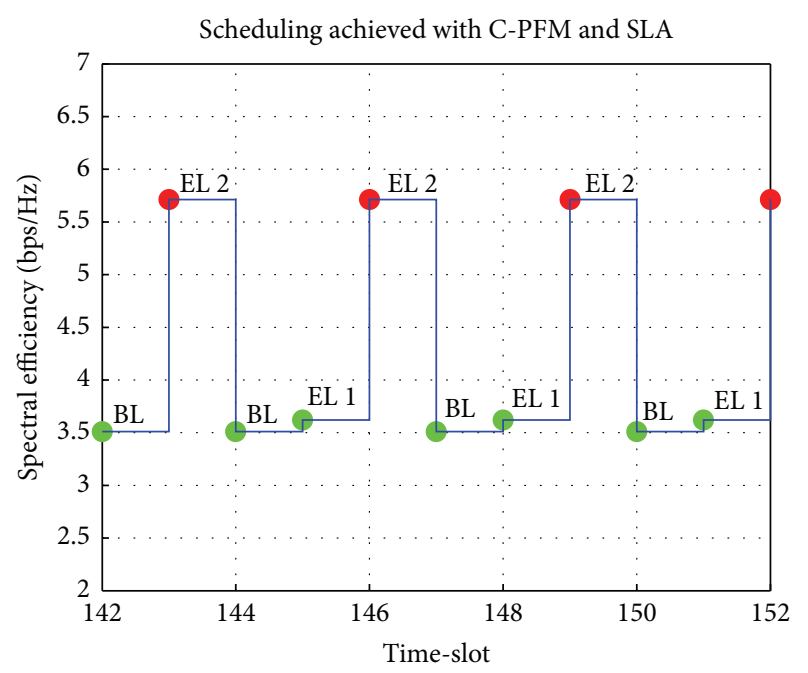

FIgURE 9: Spectral efficiency transmitted in each time-slot in the C-PFM scheme for UTs distribution in Spain and considering a minimum SLA. Green dots signal time-slots where the multilink with NC capability is not enabled. Red dots signal time-slots where the multilink with NC capability is enabled.

to the UT while still providing significant gains (21\%) even under rain conditions (15\%). In addition, we have suggested, for some relevant multicast applications, proper techniques at the upper layers to deal with the different amounts of data delivered to each UT. Finally, the gains achieved by our scheme do not require the use of additional resources but only enable UTs accessing orthogonal transmissions already in use by UTs in other beams.

As future line of research, we would like to investigate and analyze the performance of the proposed scheme in a mobile environment where the election of a suitable modulation and codification becomes more challenging since UTs are subject to fast channel variations. Moreover, our intraservice policy could be combined with interservice policies in order to meet the different requirements demanded by multicast services.

\section{Conflict of Interests}

The authors declare that there is no conflict of interests regarding the publication of this paper.

\section{References}

[1] B. Ahlgren, C. Dannewitz, C. Imbrenda, D. Kutscher, and B. Ohlman, "A survey of information-centric networking," IEEE Communications Magazine, vol. 50, no. 7, pp. 26-36, 2012.

[2] H. Fenech, Amos, Tomatis, and Soumpholphkakdy, "KA-SAT and future HTS systems," in Proceedings of the 14th IEEE International Vacuum Electronics Conference (IVEC '13), pp. 1-2, IEEE, Paris, France, May 2013.

[3] S. H. Blumenthal, "Medium earth orbit ka band satellite communications system," in Proceedings of the IEEE Military Communications Conference (MILCOM '13), pp. 273-277, IEEE, San Diego, Calif, USA, November 2013.
[4] D. Christopoulos, S. Chatzinotas, and B. Ottersten, "User scheduling for coordinated dual satellite systems with linear precoding," in Proceedings of the IEEE International Conference on Communications (ICC '13), pp. 4498-4503, June 2013.

[5] S. K. Sharma, S. Chatzinotas, and B. Ottersten, "Cognitive beamhopping for spectral coexistence of multibeam satellites," International Journal of Satellite Communications and Networking, vol. 33, no. 1, pp. 69-91, 2014.

[6] ETSI: EN 302 307, Digital Video Broadcasting (DVB); Second generation framing structure, channel coding and modulation systems for Broadcasting, Interactive Services, News Gathering and other broadband satellite applications (DVB-S2), 2009.

[7] DVB Document A83-2, Digital Video Broadcasting (DVB), Second generation framing structure, channel coding and modulation systems for Broadcasting, Interactive Services, News Gathering and other broadband satellite applications. Part II: S2-Extensions (DVB-S2X), 2014.

[8] M. A. Vázquez-Castro and N. Alagha, "Multi-link reception multibeam satellite system model," in Proceedings of the 6th Advanced Satellite Multimedia Systems Conference (ASMS) and 12th Signal Processing for Space Communications Workshop (SPSC '12), pp. 132-138, Baiona, Spain, September 2012.

[9] X. Tang and H. Yang, "Effect of user distribution on the capacity of cellular networks," in Proceedings of the National Conference on Information Technology and Computer Science (CITCS '12), pp. 370-373, 2012.

[10] C. Qian, S. Zhang, and W. Zhou, "Traffic-based dynamic beam coverage adjustment in satellite mobile communication," in Proceedings of the 6th International Conference on Wireless Communications and Signal Processing (WCSP '14), pp. 1-6, October 2014.

[11] A. Tunpan and M. S. Corson, "Bulk data multicast rate scheduling for hybrid heterogeneous satellite-terrestrial networks," in Proceedings of the 5th IEEE Symposium on Computers and Communications (ISCC '00), pp. 238-244, July 2000.

[12] D. P. Fernández and M. A. V. Castro, "Multicast transmission optimization over hybrid DVB-SH systems," in Proceedings of the IEEE 69th Vehicular Technology Conference (VTC Spring '09), pp. 1-5, IEEE, Barcelona, Spain, April 2009.

[13] D. P. Fernández and M. A. Vázquez Castro, "Cross-layer rate allocation of multicast transmission over hybrid DVB-SH," in Proceedings of the 2nd International Workshop on Cross Layer Design (IWCLD '09), pp. 1-5, June 2009.

[14] A. Sali, G. Acar, B. Evans, and G. Giambene, "A comparison of multicast adaptive techniques in reliable delivery over GEO satellite networks," in Proceedings of the IEEE Vehicular Technology Conference (VTC Spring '09), pp. 1-5, April 2009.

[15] D. Pradas and M. A. Vázquez-Castro, "NUM-based fair ratedelay balancing for layered video multicasting over adaptive satellite networks," IEEE Journal on Selected Areas in Communications, vol. 29, no. 5, pp. 969-978, 2011.

[16] F. Vieira, S. Shintre, and J. Barros, "How feasible is network coding in current satellite systems?" in Proceedings of the 5th Advanced Satellite Multimedia Systems Conference (ASMA) and the 11th Signal Processing for Space Communications Workshop (SPSC '10), pp. 31-37, Cagliari, Italy, September 2010.

[17] F. Vieira, D. Lucani, and N. Alagha, "Load-aware soft-handovers for multibeam satellites: a network coding perspective," in Proceedings of the ASMS Conference and SPSC Workshop, pp. 189-196, 2012.

[18] F. Vieira, D. E. Lucani, and N. Alagha, "Codes and balances: multibeam satellite load balancing with coded packets," in 
Proceedings of the IEEE International Conference on Communications (ICC '12), pp. 3316-3321, Ottawa, Canada, June 2012.

[19] R. Alegre-Godoy, N. Alagha, and M. A. Vázquez-Castro, "Offered capacity optimization mechanisms for multi-beam satellite systems," in Proceedings of the IEEE International Conference on Communications (ICC '12), pp. 3180-3184, June 2012.

[20] R. Alegre-Godoy, S. Gheorghiu, N. Alagha, and M. A. VázquezCastro, "Multicasting optimization methods for multi-beam satellite systems using network coding," in Proceedings of the AIAA International Communications Satellite Systems Conference (ICSSC '11), pp. 1-11, 2011.

[21] C. H. Koh and Y. Y. Kim, "A proportional fair scheduling for multicast services in wireless cellular networks," in Proceedings of the IEEE 64th Vehicular Technology Conference (VTC-Fall '06), pp. 1-5, Montreal, Canada, September 2006.

[22] W. L. Cook, D. D. Opiekun, and L. R. Karl, "Generation and display of satellite antenna patterns," COMSAT Technical Review, vol. 19, no. 2, pp. 259-297, 1989.

[23] Z. Huang, X. Yin, X. Wang, J. Zhao, and X. Xue, "CODED IP: on the feasibility of IP-layer network coding," in Proceedings of the 17th International Conference on Computer Communications and Networks (ICCCN '08), pp. 1-6, IEEE, August 2008.

[24] M. Newton and J. Thompson, "Classification and generation of non-uniform user distributions for cellular multi-hop networks," in Proceedings of the IEEE International Conference on Communications (ICC '06), pp. 4549-4553, July 2006.

[25] B. C. H. Holbrook and B. Haberman, "Using internet group management protocol version 3 (IGMPv3) and multicast listener discovery protocol version 2 (MLDv2) for source-specific multicast," RFC 4604, 2006.

[26] ETSI, "Digital video broadcasting (DVB); system specifications for satellite services to handheld devices (SH) below $3 \mathrm{GHz}$," ETSI TS 102585 V1.2.1 (2011-09), European Telecommunications Standards Institute, 2011.

[27] N. Capela and S. Sargento, "Optimizing network performance with multihoming and network coding," in Proceedings of the IEEE Globecom Workshops (GC Wkshps '12), pp. 210-215, IEEE, December 2012.

[28] A. Paramanathan, M. Pedersen, D. Lucani, F. Fitzek, and M. Katz, "Lean and mean: network coding for commercial devices," IEEE Wireless Communications, vol. 20, no. 5, pp. 54-61, 2013.

[29] S. Lee and W. W. Ro, "Accelerated network coding with dynamic stream decomposition on graphics processing unit," The Computer Journal, vol. 55, no. 1, pp. 21-34, 2012.

[30] M. Á. Vázquez-Castro and F. Vieira, "DVB-S2 full cross-layer design for QoS provision," IEEE Communications Magazine, vol. 50, no. 1, pp. 128-135, 2012.

[31] E. Rendon-Morales, J. Mata-Díaz, J. Alins, J. L. Muñoz, and O. Esparza, "Cross-layer packet scheduler for QoS support over digital video broadcasting-second generation broadband satellite systems," International Journal of Communication Systems, vol. 27, no. 10, pp. 2063-2082, 2014.

[32] H. Liu and F. Sun, "A QoS-oriented congestion control mechanism for satellite networks," Mathematical Problems in Engineering, vol. 2014, Article ID 487483, 13 pages, 2014.

[33] A. Gharanjik, B. Shankar, P.-D. Arapoglou, and B. Ottersten, "Multiple gateway transmit diversity in Q/V band feeder links," IEEE Transactions on Communications, vol. 63, no. 3, pp. 916926, 2015.
[34] A. Kyrgiazos and B. Evans, "Gateway diversity for Q/V feeder links: requirements, characteristics, and challenges," in Proceedings of the 7th Advanced Satellite Multimedia Systems Conference and the 13th Signal Processing for Space Communications Workshop (ASMS/SPSC '14), pp. 323-330, Livorno, Italy, September 2014.

[35] Centro Nacional de Información Geográfica (CNIG), http:// www.cnig.es/.

[36] F. A. Mogus, "Performance comparison of multiple description coding and scalable video coding," in Proceedings of the IEEE 3rd International Conference on Communication Software and Networks (ICCSN '11), pp. 452-456, May 2011.

[37] E. Ravelli, V. Melkote, T. Nanjundaswamy, and K. Rose, “Joint optimization of the perceptual core and lossless compression layers in scalable audio coding," in Proceedings of the IEEE International Conference on Acoustics, Speech, and Signal Processing (ICASSP '10), pp. 365-368, IEEE, Dallas, Tex, USA, March 2010. 


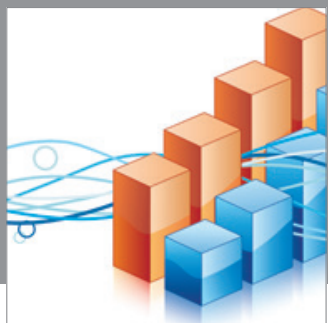

Advances in

Operations Research

mansans

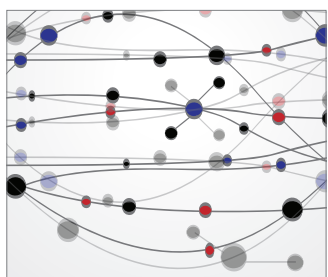

The Scientific World Journal
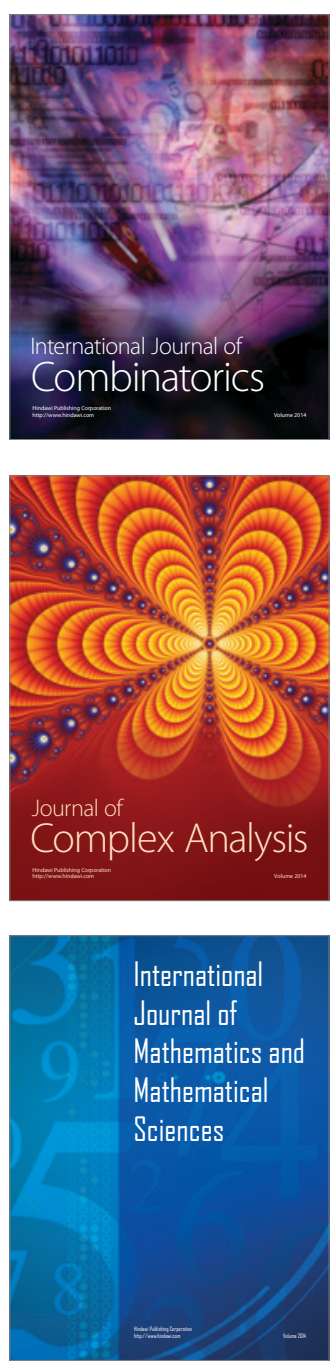
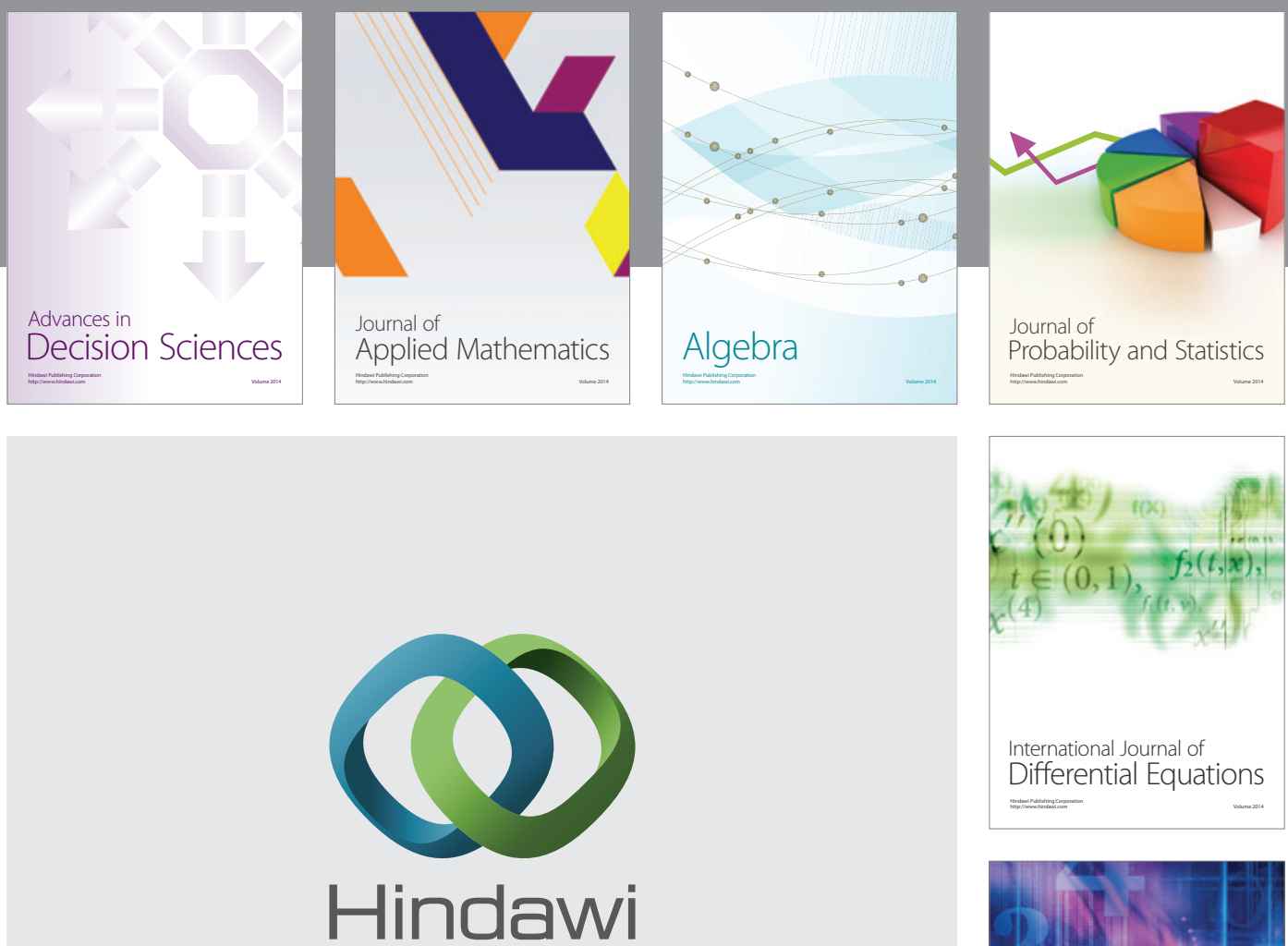

Submit your manuscripts at http://www.hindawi.com
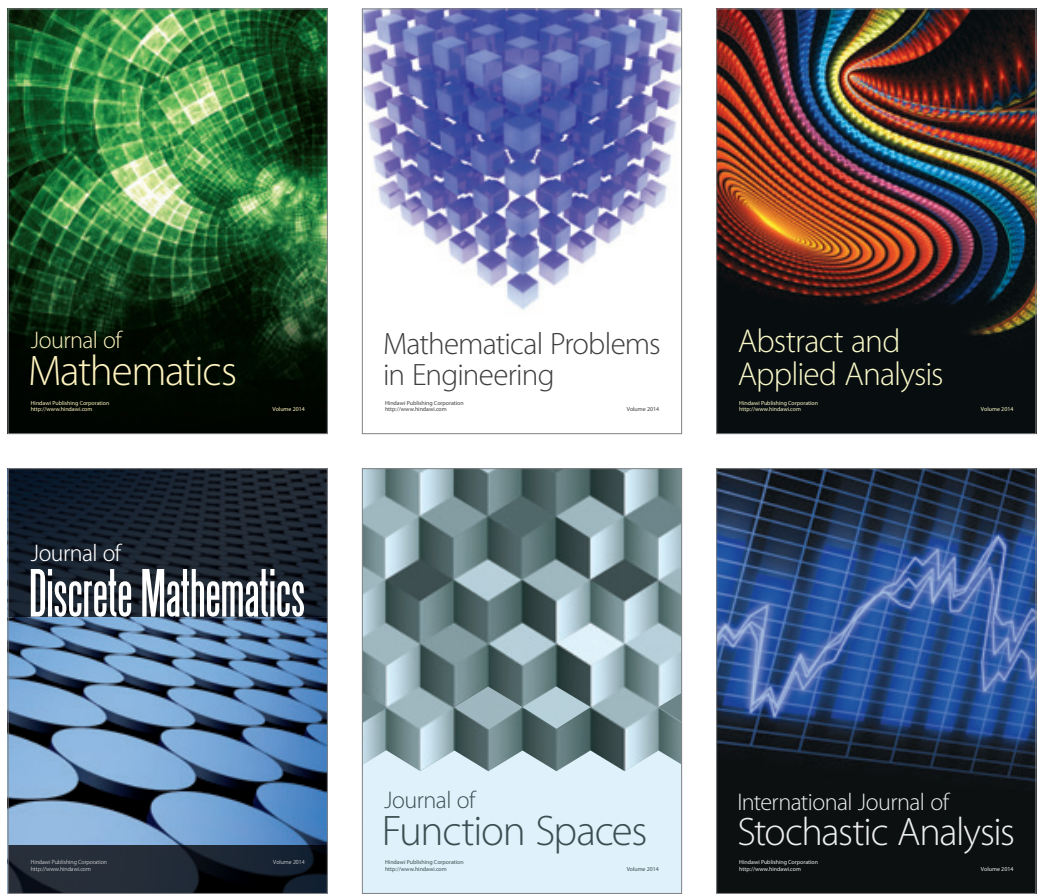

Journal of

Function Spaces

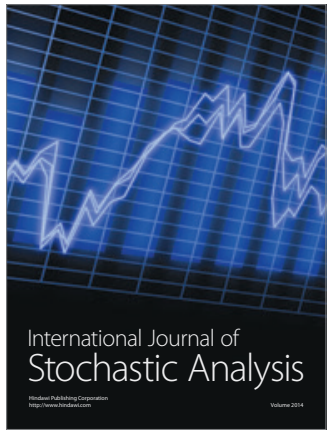

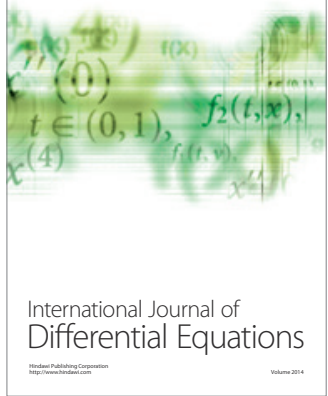
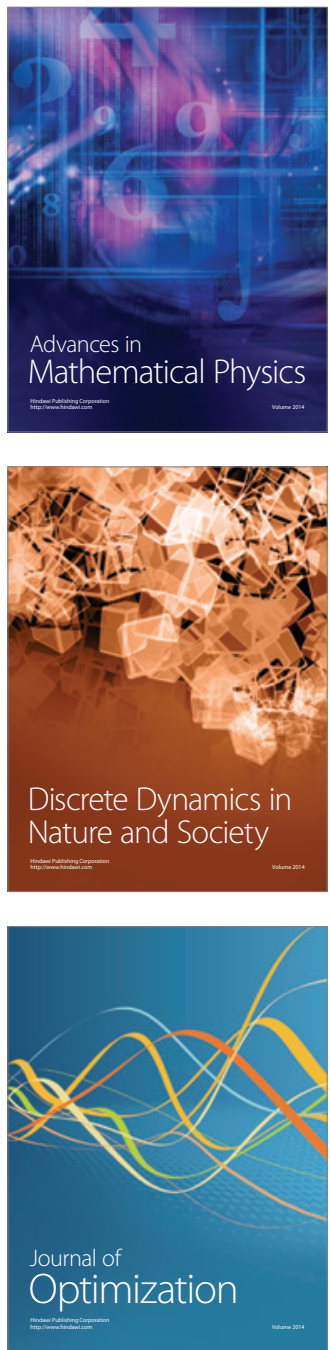